\title{
Chirurgische Therapieoptionen primärer und sekundärer Tumoren der Wirbelsäule
}

\author{
Christian W. Müller, Rebecca Stier, Markus Oszwald, Christian Krettek, Thomas Gösling
}

\section{Zusammenfassung}

Während primäre Tumoren der Wirbelsäule eher selten vorkommen, ist die Wirbelsäule der häufigste skeletale Manifestationsort für Metastasen. Abhängig von Resektabilität, Tumorentität und Neurologie kommen als chirurgische Therapieoptionen die En-bloc-Resektion, die intraläsionale Resektion, die palliative Dekompression und Stabilisierung oder die (in der Regel ebenfalls palliative) Zementaugmentation als Vertebro- oder Kyphoplastie zur Anwendung. Prognosescores können die Entscheidung erleichtern, letztlich ist jedoch eine individuelle Therapieentscheidung erforderlich, die im Kontext mit der Behandlung und den Behandlern des Primärtumors getroffen werden sollte.

\section{Surgical Treatment Options for Primary and Secondary Tumours of the Spine}

While primary tumours of the spine are rare, the spine is the most common site in the skeletal system for metastases. Depending on resectability, type of tumour and existing or pending neurological deficits, surgical options are en-bloc-resection, intralesional resection, palliative decompression and stabilisation (as a rule also palliative) or vertebro- and kyphoplasties (augmentation with bone cement). Scoring systems can help to determine the life expectancy and subsequently influence the choice of options; nevertheless each decision has to be made on an individual basis and should be the result of an interdisciplinary approach together with radiotherapists and oncologists.

\section{Einleitung}

Tumoren der Wirbelsäule stellen in besonderer Weise eine Bedrohung für Leben und Lebensqualität des Patienten dar. Zum einen gehen sie regelmäßig mit erheblicher Schmerzsymptomatik einher, zum anderen gefährden sie meistens kurz- oder mittelfristig die neurologische Integrität und die Stabilität der Wirbelsäule. Viele der Wirbelsäulentumoren erfordern eine operative Therapie, welche, zumal wenn sie nach onkologischen Prinzipien erfolgt, häufig sehr invasiv ist und somit ihrerseits die Lebensqualität einschränkt.

OP-JOURNAL 2010; 26: 208-212

(c) Georg Thieme Verlag KG Stuttgart · New York DOI http://dx.doi.org/10.1055/s-0030-1250587

\section{Primäre Tumoren der Wirbelsäule}

Der Anteil der primären Tumoren an allen Tumormanifestationen der Wirbelsäule ist mit etwa $3 \%$ relativ gering, in entsprechenden Zentren beträgt der Anteil allerdings bis zu 47\% [1]. Auch bezogen auf die Entität der primären Knochentumoren insgesamt sind Wirbelsäulentumoren mit einem Anteil von bis zu $2-10 \%$ selten. Unterschieden werden gutartige und bösartige primäre Wirbelsäulentumoren, wobei auch gutartige Tumoren lokal destruktiv wachsen oder maligne entarten können und dann entsprechend radikal behandelt werden müssen.

Häufige benigne Wirbelsäulentumoren sind das Osteoblastom, das Hämangiom, die aneurysmatische Knochenzyste und das benigne fibröse Histiozytom. Häufige maligne primäre Tumoren sind das Myelom, das Angiosarkom, das Chor-
dom/Chondrosarkom und das maligne Lymphom [1,2].

Neurologische Affektionen werden bei $30 \%$ der benignen und $70 \%$ der malignen Tumoren beschrieben [3].

\section{Sekundäre Tumoren der Wirbelsäule}

Anders als bei den primären Knochentumoren stellt die Wirbelsäule einen ausgesprochen häufigen Manifestationsort für Metastasen dar, den häufigsten des Skelettsystems und Bewegungsapparats.

Nach der offiziellen Todesursachenstatistik sterben rund $1 / 4$ der Bundesbürger an einem Tumorleiden (www.destatis. de). Es wird geschätzt, dass ca. $40 \%$ aller Krebspatienten im Verlauf Wirbelsäulenmetastasen entwickeln und ca. 10-20\% in der Folge symptomatische Einengungen des Rückenmarks [4,5]. Sensible Defizite werden in 70-80\%, Paraparesen und Paraplegien in 60\%, Störungen von Stuhlgangs- und Harnkontrolle in bis zu $77 \%$ der symptomatischen Fälle beschrieben [4]. In Autopsiestudien wurden bei $90 \%$ der an Prostatakarzinom Verstorbenen Metastasen in der Wirbelsäule gefunden, bei 70\% derer mit Mammakarzinom, bei 55\% der Patienten mit malignem Melanom, bei $45 \%$ derer mit Bronchialkarzinom [6].

Mit 70\% häufigste Lokalisation ist die Brustwirbelsäule, 20\% der Metastasen betreffen die Lendenwirbelsäule, 10\% die Halswirbelsäule, bis zu 38\% treten multilokulär auf. Als Grund für die häufige Metastasierung in die Wirbelsäule wird der langsame Blutfluss in den Wirbelkörpern angenommen [4,5].

\section{Klinische Diagnostik}

Anamnese und klinische Untersuchung sollten neben den allgemeinen und tumorspezifischen Fragestellungen v.a. die genaue neurologische Funktion erfassen. 
Leitsymptom und Grund für die Vorstellung ist in der Regel der therapieresistente Rückenschmerz, gelegentlich auch die neurologische Verschlechterung.

Insbesondere bei bekanntem Rückenschmerz anderer Genese und bislang leerer Tumoranamnese führt dann manchmal erst die neu aufgetretene Gangunfähigkeit zur weiteren Diagnostik und zur Diagnosestellung eines metastasierten Tumorleidens. Bei primär unbekanntem Primärtumor wird am häufigsten ein Bronchialkarzinom als Ursache gefunden [4]. In der Regel erfolgt die Klassifizierung des neurologischen Befunds nach Frankel [4]:

- Frankel A: komplette Paraplegie (Lähmung)

- Frankel B: komplette motorische Lähmung bei erhaltener Sensibilität

- Frankel C: erhebliche motorische Lähmung

- Frankel D: leichte motorische Lähmung

- Frankel E: Motorik und Sensibilität erhalten

\section{Bildgebung}

Die bildgebende Diagnostik erfordert grundsätzlich eine Computertomografie (CT) mit Reformationen zur Beurteilung des Ausmaßes der knöchernen Wirbeldestruktion. Die konventionelle Röntgendiagnostik kann hingegen zwar auch Hinweise auf knöcherne Destruktionen und pathologische Frakturen geben, ist aber letztlich sowohl als Screening-Methode als auch zur Artdiagnostik des Tumors unzureichend. Bei neurologischen Ausfällen und zur weiteren Artdiagnostik, aber auch zur Beurteilung von extraossären Tumoranteilen, zumal intraduralen, ist meistens eine Magnetresonanztomografie (MRT) angezeigt. Ausnahmsweise, bei Kontraindikation gegen ein MRT, kommt noch die CTMyelografie zum Einsatz, während die Szintigrafie bei hoher Sensitivität, aber geringer Spezifität v.a. zur Ausschlussdiagnostik geeignet ist, aber zunehmend seltener zum Einsatz kommt [7].

\section{Indikationsstellung zur Operation}

Primäre Wirbelsäulentumoren und solitäre Metastasen können in einigen Fällen unter einem kurativen Ansatz operiert werden (En-bloc-Resektion). Hier ergibt sich die Indikation aus der Chance auf eine definitive Heilung.
Daneben sind die wichtigsten Indikationen zur Operation:

- Schmerzen, die schmerztherapeutisch nicht adäquat therapierbar sind - akut drohende oder bereits bestehende neurologische Ausfälle

- drohende oder manifeste Instabilität und Deformierung der Wirbelsäule - Diagnosesicherung

Die Wahl des operativen Verfahrens muss sowohl der Tumorentität und der lokalen Situation an der Wirbelsäule als auch dem Allgemeinzustand des Patienten und seiner Lebenserwartung Rechnung tragen. Als Kriterien für eine Instabilität wurden, aufbauend auf dem 3-Säulen-Konzept von Denis für die posttraumatische Instabilität nach Verletzungen, beschrieben [4]:

- Destruktion der vorderen und mitt-

leren Säule oder Kollaps von mehr als $50 \%$ der Wirbelkörperhöhe

- Beteiligung der mittleren und hinteren Säule

- Beteiligung derselben Säulen in benachbarten Wirbelkörpern

- iatrogene Destabilisierung durch Laminektomie oder Resektion von mehr als $50 \%$ des Wirbelkörper

\section{Prognosesysteme}

Verschiedene Scores sind entwickelt worden, um eine Abschätzung der Lebenserwartung und eine Empfehlung zu einem Therapieverfahren zu erleichtern. Am weitesten verbreitet sind die Scores von Tomita und Tokuhashi. Der TomitaScore berücksichtigt allein Charakteristika des Tumors und vergibt für langsam, moderat oder schnell wachsenden Primärtumor 1, 2 oder 4 Punkte, für behandelbare oder nicht behandelbare viszerale Metastasen 2 bzw. 4 Punkte und für solitäre oder multiple Knochenmetastasen werden 1 bzw. 2 Punkte vergeben. Aus der Summe wird dann folgende Therapieempfehlung abgeleitet [8]:

- weite oder marginale Exzision bei 2-3 Punkten

- marginale oder intraläsionale Entfernung bei 4-5 Punkten

- palliative Operation, d.h. Laminektomie und Stabilisierung bei 7-8 Punkten

- konservativ bei 8-10 Punkten

Tokuhashi hingegen stellte 1990 ein Prognosesystem vor, das auch den Allgemeinzustand des Patienten und die neurologische Symptomatik einbezog [9]. Dieses System wurde 2005 noch einmal revidiert, insbesondere wurde stär- ker nach dem Primärtumor differenziert (Tab. 1) [10]. Abgeleitet von der erwarteten Lebenserwartung wurde die weite Exzision bei guter Lebenserwartung (>12 Monate, 12-15 Punkte), die palliative Operation bei intermediärer Lebenserwartung (9-11 Punkte) und konservative Therapie oder palliative Operation bei schlechter Prognose ( $<6$ Monate; 0 8 Punkte) empfohlen [10]. Beide Prognosesysteme sind jedoch immer wieder auch kritisiert worden, weil sie zum einen teils auf retrospektiv gewonnenen Daten beruhen, zum anderen Fortschritte in der Therapie des Primärtumors der letzten Jahre unberücksichtigt bleiben.

\section{Operationsverfahren}

Trotz Prognosesystemen muss die Therapieentscheidung letztlich individuell gestellt werden. Nach der Radikalität und Invasivität sind zu unterscheiden:

- die En-bloc-Resektion oder Wide-Exzision mit dem Ziel, den Tumor in einem Stück zu entfernen im Sinne einer R0-Resektion

- das Tumor-Debulking oder die Tumormassenreduktion mit dem Ziel einer weistestmöglichen Entfernung des Tumors (R1- oder R2-Resektion)

- die dorsale Dekompression und transpedikuläre Stabilisierung der Wirbelsäule als palliatives Verfahren ohne Tumorresektion bei Instabilität und drohender oder bestehender Spinalkanaleinengung

- die perkutane Zementaugmentation zur Stabilisierung, Histologiegewinnung und Schmerztherapie bei multipler Metastasierung ohne drohende neurologische Komplikationen

\section{En-bloc-Resektion}

En-bloc-Resektionen kommen bei guter Gesamtprognose und kurativem Ansatz infrage. Die operative Strategie muss die strikte Vermeidung einer Eröffnung des Tumors bei gleichzeitiger Schonung des Rückenmarks erreichen. Abb. 1 zeigt das Beispiel einer En-bloc-Resektion. Bei der 71-jährigen Patientin war 3 Jahre zuvor ein Mammakarzinom diagnostiziert worden. Es erfolgte damals die Brustamputation und anschließende Chemotherapie. Aktuell war nun in der Verlaufskontrolle eine Lebermetastase aufgefallen. Die erneute Staging-Diagnostik zeigte eine große Osteolyse des 8. Brustwirbelkörpers, einseitig bis in den Pedikel reichend. Schmerzen bestanden im Bereich der Brustwirbelsäule nicht, jedoch Kribbelparästhesien der Zehen rechts. Die Fein- 
Tab. 1 Der revidierte Prognosescore nach Tokuhashi [10].

\begin{tabular}{lll} 
Charakteristika & & Punkte \\
\hline Allgemein- & schlecht (10-40\%) & 0 \\
zustand (Kar- & mäßig (50-70\%) & 1 \\
nofsky-Index) & gut (80-100\%) & 2 \\
\hline Anzahl extraspi- & $\geq 3$ & 0 \\
naler Knochen- & 1,2 & 1 \\
metastasen & 0 & 2 \\
\hline Anzahl Metas- & $\geq 3$ & 0 \\
tasen in der & 2 & 1 \\
Wirbelsäule & 1 & 2 \\
\hline Organ- & nicht entfernbar & 0 \\
metastasen & entfernbar & 1 \\
& keine Metastasen & 2 \\
\hline Lokalisation des & Lunge, Osteosarkom, Magen, Blase, Speiseröhre, Pankreas & 0 \\
Primärtumors & Leber, Gallenblase oder unbekannt & 1 \\
& anderer Tumor & 2 \\
& Niere, Uterus & 3 \\
& Rektum & 4 \\
& Schilddrüse, Prostata, Mamma, Karzinoid & 5 \\
\hline Lähmung & komplett (Frankel A oder B) & 0 \\
& inkomplett (Frankel C oder D) & 1 \\
\hline gesamt & keine & 2 \\
\hline vorhergesagte & Maximum & 15 \\
erwartung & 9-8 Punkte & $<6$ Monate \\
\hline & 12-15 Punkte & $>12$ Monate \\
\hline
\end{tabular}

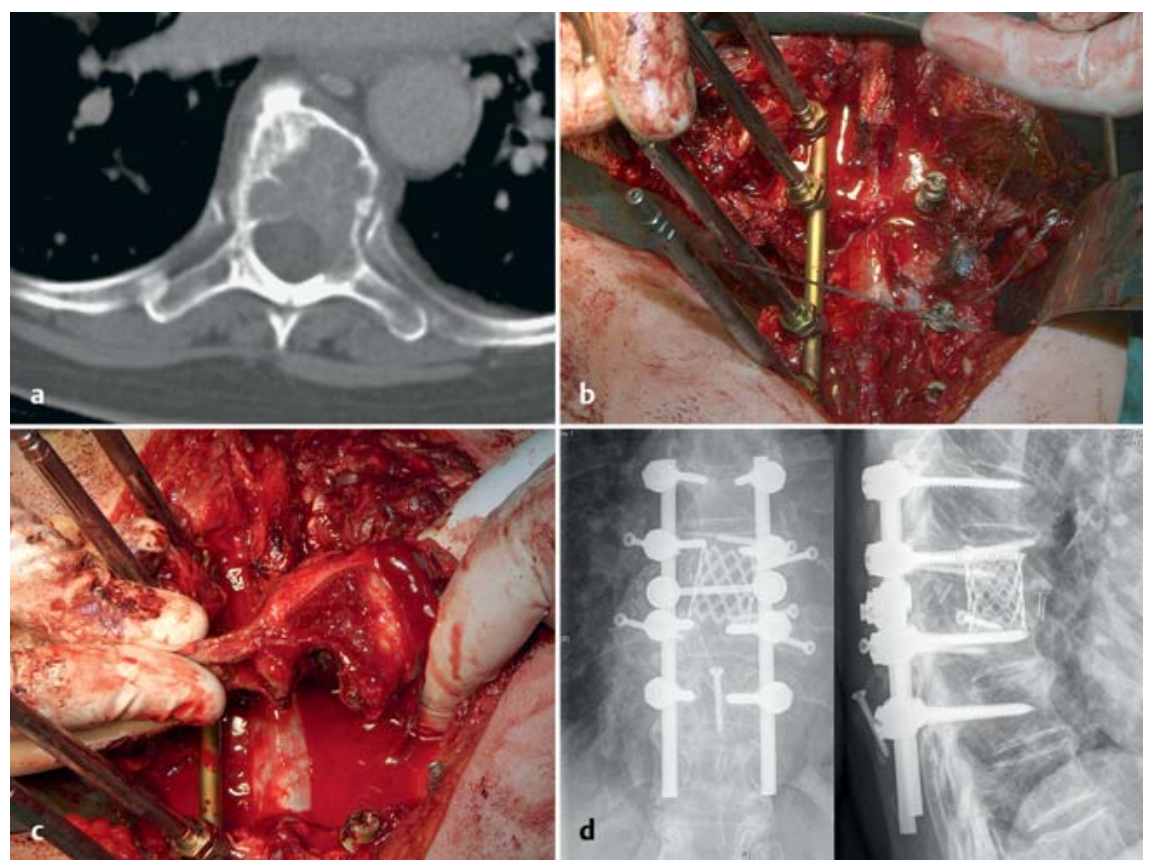

Abb. 1 a bis $\mathbf{d}$ En-bloc-Resektion bei Metastase eines Mammakarzinoms in BWK 8 (a, b präoperatives $\mathrm{CT}$ und intraoperativer Situs; c, $\mathbf{d}$ En-bloc-Resektat und postoperatives Röntgenbild, siehe Text).

schicht-CT zeigte einen vollständig osteolytischen Wirbelkörper mit Ausbreitung der Osteolyse in den linken Pedikel sowie in den linken Processus articularis superior und den linken Processus articularis inferior (Abb.1 a). Bei relativ gutem Allgemeinzustand und günstiger Prognose (Tomita-Score 3, Tokuhashi-
Score 13) wurde die Indikation zur Enbloc-Resektion des 8 . BWK, dorsaler Instrumentierung BWK 6/7 auf BWK 9/10, Spondylodese BWK 7 auf BWK 9 und Wirbelkörperersatz durch Harms-Cage gestellt.

Intraoperativ erfolgte zunächst über einen dorsomedianen Zugang die Darstellung der Dornfortsätze und Laminae BWK 5-12 und die transpedikuläre Instrumentierung BWK 6, 7, 9 und 10, dann nach Freilegen der Rippen bds. auf $12 \mathrm{~cm}$ Länge die linksseitige epipleurale Präparation und Lösung der Pleura von der 7. und 8. Rippe (Abb.1 b). Durchtrennung der 8. Rippe $2 \mathrm{~cm}$ lateral des Proc. transversus und ca. $6 \mathrm{~cm}$ weiter lateral. Durchtrennung der 7. Rippe im Bereich des Proc. transversus und ca. $8 \mathrm{~cm}$ weiter lateral. Nun Präperation lateral des Wirbelkörpers und Lösung der Aorta. Laminektomie BWK 7-9. Mobilisation des Duralsacks auf Höhe BWK 8, Durchtrennung der rechtsseitigen Wurzel BWK 8. Osteotomie im Bereich BWK 7 mit GigliSäge. Verbinden der Pedikelschrauben rechtsseitig. Osteotomie in selbiger Weise BWK 8 und 9. Anschließend nach vollständiger Mobilisierung der Metastase Herausdrehen des Resektats um den Duralsack herum nach dorsal (Abb.1c). Einbringen einen Harms-Körbchens. Einbringen der Längsstangen links. Anschrauben der resezierten Rippen im Sinne einer lateralen Spondylodese. Einbringen eines Querverbinders.

Die Metastase konnte vollständig entfernt werden. Im Anschluss der Operation erfolgte eine lokale Nachbestrahlung. Zur 6-Monats-Kontrolle war die Patientin komplett beschwerdefrei (VAS 0/10) ohne neurologische Defizite. Abb.1d zeigt die nativ-radiologische Verlaufskontrolle.

\section{Intraläsionale Resektion/ Tumordebulking}

Abb. 2 zeigt den Fall eines 6-jährigen Jungen mit unklaren Beschwerden in der HWS seit wenigen Monaten. Die primär angefertigte Röntgenkontrolle zeigte eine unklare zystische Struktur im HWK 2. Zur genaueren Diagnostik wurden eine Computertomografie und eine MRT angefertigt, welche eine Beteiligung des Dens axis und der Pedikel des HWK 2 bis in die Lamina darstellten (Abb. 2a-c). Radiologisch bestand der Verdacht auf eine aneurysmatische Knochenzyste. Bei erheblicher Schwächung der Kortikalis stellten wir die Indikation zur Stabilisa- 


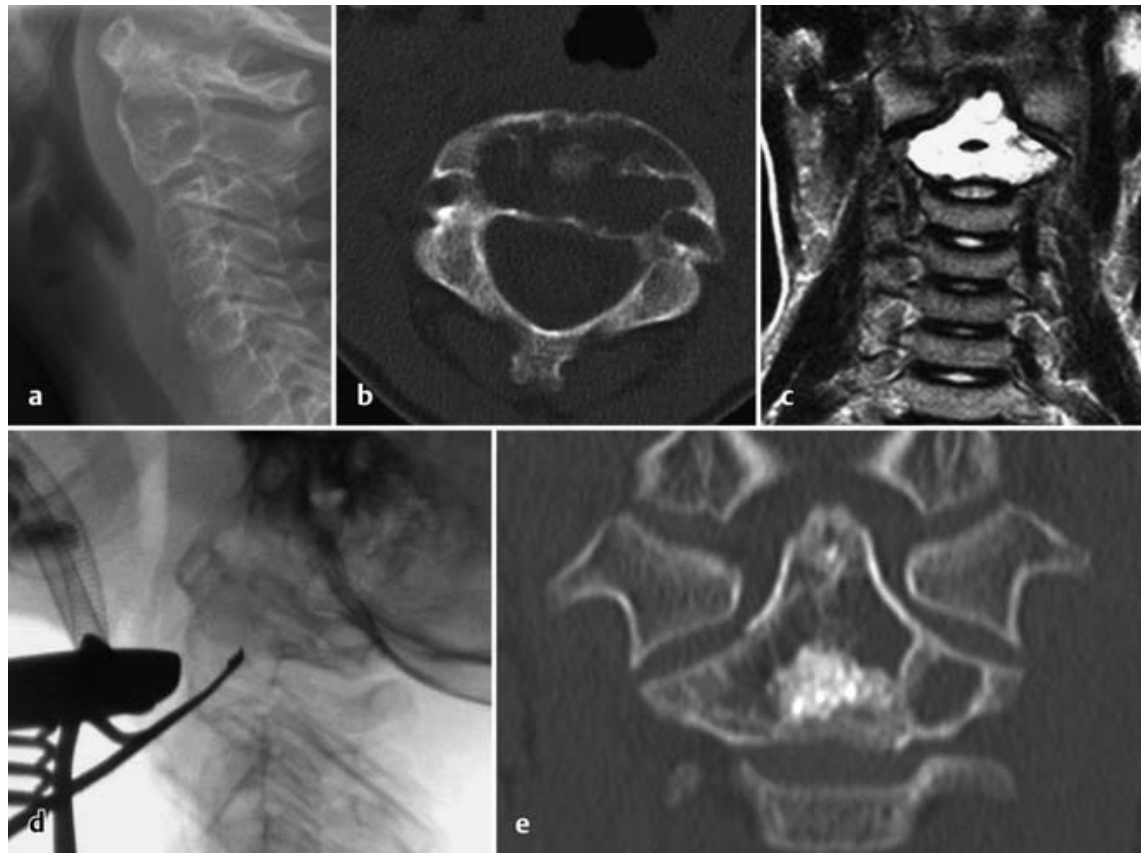

Abb. 2a bis e Intraläsionale Resektion einer aneurysmatischen Knochenzyste in HWK 2 bei einem 6-Jährigen (siehe Text).
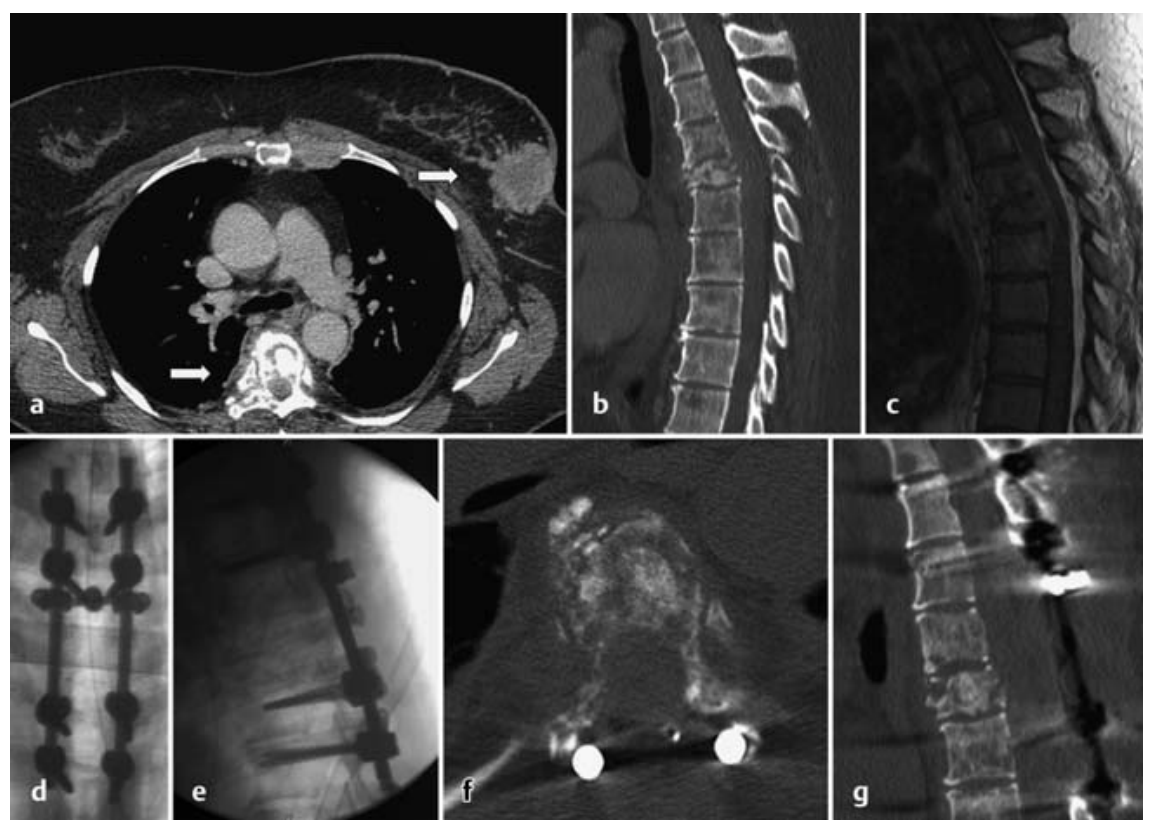

Abb. $\mathbf{3}$ a bis $\mathbf{g}$ Laminektomie und dorsale Instrumentierung bei Metastase eines zuvor nicht bekannten Mammakarzinoms mit ausgedehnten epiduralen Anteilen und manifesten neurologischen Ausfällen (a, b: Staging-CT; c: MRT; d, e: intraoperatives Röntgen; f, g: postoperatives CT).

tion. Auf eine Embolisation, die sich insbesondere bei der intraläsionalen Resektion regelmäßig empfiehlt, wurde hier aufgrund des hohen Risikos für einen Insult verzichtet, sodass man sich zu einer offenen Kürettage und Spongiosaplastik entschloss. Die Operation erfolgte in Rückenlage. Der Zugang erfolgte über einen ca. $3 \mathrm{~cm}$ langen Hautschnitt am vorderen Rand des M. sternocleidomastoideus rechtsseitig. Nach Darstellungen des 2.
Die Histologie bestätigte die Verdachtsdiagnose einer aneurysmatischen Knochenzyste mit erheblichem Knochenumbau und einer chronischen Entzündungsreaktion. Der Patient zeigte klinisch außer einer zugangsbedingten Schluckstörung und dezenter Heiserkeit keine neurologischen Ausfälle. Nach 8 Wochen Immobilisation im Miami-J-Kragen konnte bei zunehmender Beschwerdefreiheit zum Tragen einer weichen Halskrawatte übergegangen werden. Nach 6 Monaten war der Patient vollständig beschwerdefrei und erreichte das volle Bewegungsausmaß in der HWS. Radiologisch zeigte sich im CT kein Anhalt für ein Rezidiv bei unverändert einliegendem Knochenzement (Abb. 3 g).

\section{Dekompression und Stabilisierung}

Abb. 3 zeigt den Fall einer 51-jährigen Patientin, die sich wegen zunehmender Schwäche der Beine in einer Klinik vorstellte. Die MRT zeigte eine maligne Wirbelsinterung BWK 5 mit epiduralen intraspinalen Tumoranteilen bei klinischem Verdacht auf ein Mammakarzinom linksseitig. Bei zuletzt schnell progredienter Neurologie bis zur Gehunfähigkeit und inkompletter Paraparese erfolgte die Zuverlegung. Das unmittelbar durchgeführte Staging-CT zeigte ausgedehnte Destruktionen und Osteolysen BWK 5 und BWK 4, daneben als wahrscheinlichen Primärtumor die Raumforderung in der linken Brust (siehe Pfeile). Es erfolgte die notfallmäßige Dekompression BWK 3-6 und die dorsale Instrumentierung BWK 2/3-6/7 und dorsale Spondylodese BWK 2-7. Die weitere Diagnostik zeigte multiple weitere Metastasen in LWS, Becken, Sakrum, Oberschenkel. Klinisch waren die neurologischen Ausfälle postoperativ nach einigen Tagen dann deutlich rückläufig, die Patientin konnte mit Einschränkungen wieder mobilisiert und einer Chemotherapie zugeführt werden.

\section{Perkutane Zementaugmentierung}

Vertebroplastie und Kyphoplastie stellen in Fällen von schmerzhaften Wirbelsäulenmetastasen eine minimalinvasive Therapieoption dar, wenn ein kurativer Ansatz ausscheidet und keine neurologische Beeinträchtigung droht. Die Operationstechnik ist im OP-Journal April 2009 ausführlich beschrieben [11]. Häufig findet die Zementaugmentierung beim Plasmozytom der Wirbelsäule Anwendung. Abb. 4 zeigt prä- (a) und postoperative (b) Röntgenbilder eines 50-jährigen Pa- 


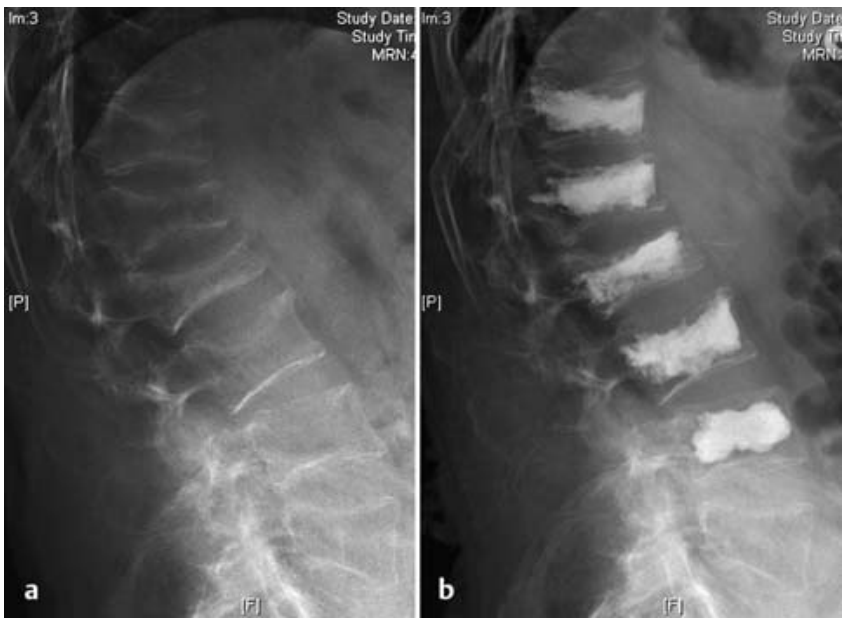

tienten, der sich bei mit erheblichen, zuletzt immobilisierenden Rückenschmerzen seit wenigen Monaten - aktuellem VAS 10/10 - und Gewichtsverlust von $5 \mathrm{~kg}$ vorstellte. Die klinische, radiologische und laborchemische Diagnostik ergab die Diagnose eines Plasmozytoms mit pathologischen Wirbelkörperfrakturen BWK 12 und LWK 1 bis 4. Parallel zur Einleitung einer Chemotherapie erfolgte die Kyphoplastie BWK 12 bis LWK 4 in einer Sitzung. Postoperativ war der Patient unmittelbar erheblich beschwerdegebessert und konnte wieder voll mobilisiert werden. Die postoperative Röntgenkontrolle zeigt auch eine teilweise Wiederaufrichtung der eingebrochenen Wirbelkörper und eine Reduktion der kyphotischen Fehlstellung um $10^{\circ}$.

Eine zusätzliche Option stellt möglicherweise in Zukunft die „Kypho-IORT“ dar: Hier wird über einen transpedikulären Zugang zunächst intraoperativ eine Hochdosisradiatio im Wirbelkörper durchgeführt, anschließend über denselben Zugang und die Gegenseite eine Kyphoplastie zur Zementaugmentation [12]. Damit könnte auf minimalinvasivem Wege eine der intraläsionalen Resektion vergleichbare Effektivität erreicht werden.
Abb. 4 a und $b$ Ballonkyphoplastie BWK 12 (a: präoperativ) und LWK 1-4 (b: postoperativ) bei pathologischen Frakturen infolge Plasmozytom (siehe Text).

\section{Fazit}

Tumoren der Wirbelsäule stellen ein sehr heterogenes Krankheitsbild dar. In der klinischen Praxis handelt es sich dabei am häufigsten um Patienten mit Metastasen in der Wirbelsäule; durch die verbesserte Kontrolle der Grundleiden treffen wir diese zunehmend an. Während bei primären Tumoren abhängig von Ausdehnung und Ausbreitung grundsätzlich die Möglichkeit einer R0-Resektion geprüft werden muss und gegen mögliche neurologische Kompromittierung abgewogen werden muss, stehen bei der häufigeren Behandlung von Patienten mit metastatischen Absiedlungen in der Wirbelsäule die Bewahrung der Stabilität, die Verhütung von neurologischen Beeinträchtigungen und die Kontrolle der Schmerzen im Vordergrund, da ein kurativer Ansatz hier nur selten möglich ist. Prognosescores stellen eine gewisse Entscheidungshilfe dar, letztlich muss jedoch auch in der metastasierten Situation eine patientengerechte Entscheidung für den Einzelfall getroffen werden.

\section{Literatur}

${ }^{1}$ Harms J, Melcher RP. Onkologische Chirurgie der Wirbelsäule. Der Chirurg 2008; 79: 927298, 30-36

2 Immenkamp M, Härle A. Geschwülste der Wirbelsäule - Knochentumore. In: Witt AN, Rettig H, Schlegel KF, Hrsg. Orthopädie in Praxis und Klinik. Stuttgart: Thieme; 1994

${ }^{3}$ Krämer J. Orthopädie und Orthopädische Chirurgie: Wirbelsäule, Thorax. 1. Aufl. Stuttgart: Thieme; 2004

4 Schaser KD, Melcher I, Mittlmeier T et al. Chirurgisches Management von Wirbelsäulenmetastasen. Der Unfallchirurg 2007; 110: 137-159

${ }^{5}$ Klimo Jr P. Schmidt MH. Surgical management of spinal metastases. The Oncologist 2004; 9: 188-196

6 Wong DA, Fornasier VL, MacNab I. Spinal metastases: the obvious, the occult, and the impostors. Spine (Phila Pa 1976) 1990; 15: 1-4

7 Ahlhelm F], Fries P, Nabhan A et al. [Spinal tumors]. Der Radiologe 2010; 50: 165-178; quiz 79-80

8 Tomita K, Kawahara N, Kobayashi T et al. Surgical strategy for spinal metastases. Spine (Phila Pa 1976) 2001; 26: 298-306

9 Tokuhashi Y, Matsuzaki H, Toriyama S et al. Scoring system for the preoperative evaluation of metastatic spine tumor prognosis. Spine (Phila Pa 1976) 1990; 15: 1110-1113

10 Tokuhashi Y, Matsuzaki H, Oda H et al. A revised scoring system for preoperative evaluation of metastatic spine tumor prognosis Spine (Phila Pa 1976) 2005; 30: 2186-2191

${ }_{11}$ Müller CW, Krettek C. Vertebroplastie und Kyphoplastie bei osteoporotischen Wirbelkörperfrakturen: Indikationen, Technik, Ergebnisse und Komplikationen. OP-Journal 2009; 25: 28-32

12 Wenz F, Schneider F, Neumaier C et al. KyphoIORT - a novel approach of intraoperative radiotherapy during kyphoplasty for vertebral metastases. Radiat Oncol 2010; 5: 11

\section{Dr. med. Christian W. Müller Oberarzt \\ Dr. med. Rebecca Stier \\ Assistenzärztin \\ Dr. med. Markus Oszwald \\ Facharzt \\ Prof. Dr. med. Christian Krettek \\ Direktor der Klinik \\ Prof. Dr. med. Thomas Gösling \\ Ltd. Oberarzt der Klinik}

Unfallchirurgische Klinik (Ärztl. Direktor: Prof. Dr. med. Chr. Krettek, FRACS) Medizinische Hochschule Hannover Carl-Neuberg-Straße 1 30625 Hannover

mueller.christian@mh-hannover.de www.mhh-unfallchirurgie.de 